\title{
EXPERIMENTAL AND NUMERICAL STUDY OF CONTROLLED FLUTTER TESTING IN A LINEAR TURBINE BLADE CASCADE
}

\author{
VÁclav Sláma ${ }^{a, *}$, BartoloměJ Rudas $^{a}$, Petr ERet $^{b}$, \\ Volodymyr TsymbalyuK ${ }^{b}$, JiŘí IrA $^{c}$, Aleš MACAlKa $^{c}$, LOREnzo Pinelli ${ }^{d}$, \\ Federico Vanti ${ }^{d}$, Andrea Arnone ${ }^{d}$, Antonio Alfio lo Balbo ${ }^{e}$ \\ ${ }^{a}$ Doosan Skoda Power s.r.o., Tylova 1/57, Pilsen 30128, Czech Republic \\ ${ }^{b}$ University of West Bohemia, Department of Power System Engineering, Univerzitni 8, Pilsen 30614, Czech \\ Republic \\ ${ }^{c}$ NUM solution s.r.o., U Pergamenky 1145/12, Prague 17000, Czech Republic \\ ${ }^{d}$ University of Florence, Via S.Marta 3, 50139 Florence, Italy \\ e Ansaldo Energia S.p.A., Via Nicola Lorenzi 8, 16152 Genova, Italy \\ * corresponding author: vaclav.slama@doosan.com
}

\begin{abstract}
In this paper, experimental testing of flutter and numerical simulations using a commercial code ANSYS CFX and an in-house code TRAF are performed on an oscillating linear cascade of turbine blades installed in a subsonic test rig. Bending and torsional motions of the blades are investigated in a travelling wave mode approach. In each numerical approach, a rig geometry model with a different level of complexity is used. Good agreement between the numerical simulations and experiments is achieved using both approaches and benefits and drawbacks of each technique are commented in this paper. It is demonstrated that both used computational techniques are adequate to predict turbine blade flutter. It is concluded that validated numerical tools can provide a better insight of flutter phenomena of operationally flexible steam turbine last stage blades.
\end{abstract}

KeYwords: Steam turbine, last stage blades, flutter, experiment, test rig, measurement, numerical analysis, CFD, validation, blade cascade.

\section{INTRODUCTION}

With the increase of steam turbine operational flexibility, the risk of asynchronous blade vibrations induced by flow becomes higher and higher and may lead to undesired failures of last stage blades (LSBs). For this reason, the aerodynamic and structural design of LSBs, which nowadays may be over 1 meter in length, is a critical aspect faced by designers. The need for robust numerical tools to predict flutter of blade cascades is a key aspect for the aeromechanical design of new modern LSBs [1]. A more flexible way to operate steam turbines highly increases the risk of flutter induced vibrations therefore it is essential to validate applied numerical tools. As the validation is not possible in industrial environments (real turbines) controlled flutter tests on simplified experimental models must be used. Such an experimental rig has been built at the Department of Power System Engineering at the University of West Bohemia (UWB) where the unsteady aerodynamic forces and moments in the oscillating turbine blade cascade can be investigated. The Flexturbine project (EC Horizon 2020, No. 653941) gives the opportunity to design and test flutter-resistant blades for a wide range of steam turbine operating conditions. Experimental and numerical studies in this project are fundamental for a deeper understanding of flutter occurrences, so that free flutter design rules for a next generation of flutter-resistant steam turbine blades can be drawn. As a part of this project, a subsonic test rig installed at the UWB is employed for a controlled flutter testing of last stage blade tip profiles in air flow. Unsteady aerodynamic forces and moments induced by blade cascade oscillations are measured and a work exchange between the blade and the flow is estimated. Two different numerical methods were applied for the flutter assessment of blade cascade: a commercial code ANSYS CFX and an in-house code TRAF. Numerical methods for flutter assessment have been firstly validated in low pressure environment for the aeronautical industry [2, 3]. More recently, the code validation has been extended to compressor blades and steam turbine rows [4, 5] so that the actual design process now includes an extensive use of these methods to achieve a free flutter design [6]. Such methodologies are usually classified in time-linearized, harmonic balance and non-linear approaches both in frequency and time domain and require high quality experimental data for the validation and tuning, especially in off-design conditions. The stability results are evaluated by aerodynamic work which describes the exchange of energy between blade and flow [7, 8 . 


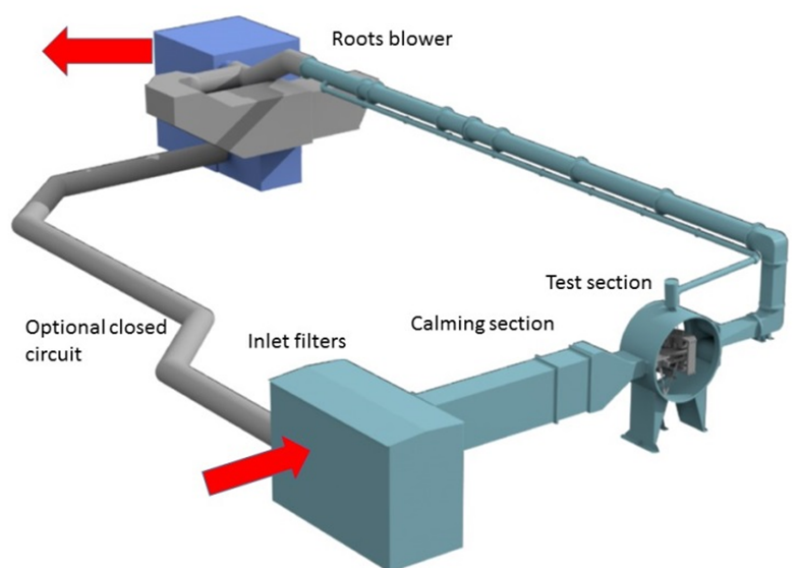

FIGURE 1. UWB test-rig, wind tunnel.

\section{TEST RIG DESCRIPTION AND MEASURED DATA ACQUISITION}

The overall view of the subsonic wind tunnel employed in experiments is shown in Fig. 1] Air flows from the atmosphere through a filter system (the inlet filters) into a smooth contraction section (the calming section) and then into a test section duct (the test section). Inside this test section, there is a linear blade cascade consisting of eight blades (Fig. 2). Inner four blades (3-6) are flexibly mounted with two degrees of freedom (the torsion and bending motion). The blade height is $80 \mathrm{~mm}$, the blade chord is $50 \mathrm{~mm}$, the maximum blade thickness is $2.25 \mathrm{~mm}$, the blade pitch is $45 \mathrm{~mm}$ and the cascade stagger angle is $72^{\circ}$. A variable angle of attack from $-15^{\circ}$ to $+15^{\circ}$ can be set and measured. Downstream of the blade cascade, the air is expanded in the drum chamber and runs through the outlet duct towards the Roots blower vacuum inlet. There are two important measurement places in the test section as can be seen in Fig. 2. The channel inlet is instrumented by a Pitot probe, a flush-mounted static port and a thermometer sensor to evaluate the inlet flow velocity and prepare boundary conditions for numerical analyses. Downstream of the blade cascade, a traversing mechanism is fitted with a Pitot-static probe to evaluate the pressure profiles (the total and static pressure profile). These profiles are important for the comparison with steady-state numerical results. BHV 5355 differential pressure transducers are used to measure all the pressures. Traverser motion is provided by a linear stepper motor. Blade motion is measured using a pair of non-contacting eddy current displacement sensors Schenck IN-085 as it is shown in Fig. 4. The bending motion can be evaluated as a mean of two signals and the torsion is proportional to the difference of signals. A simultaneously updating analog output module NI 9264 was programmed in LabVIEW to generate reference signals for eight analog controllers. A NI PXI 1042 chassis with three PXI 4472b modules has been used for a simultaneous sampling of all signals.

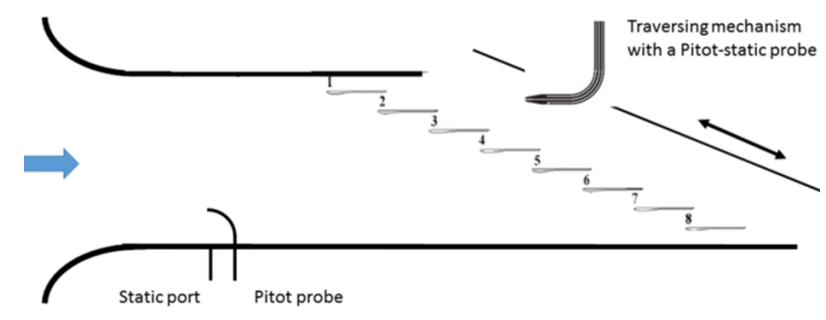

FiguRE 2. Schematic of the test section part, top view.

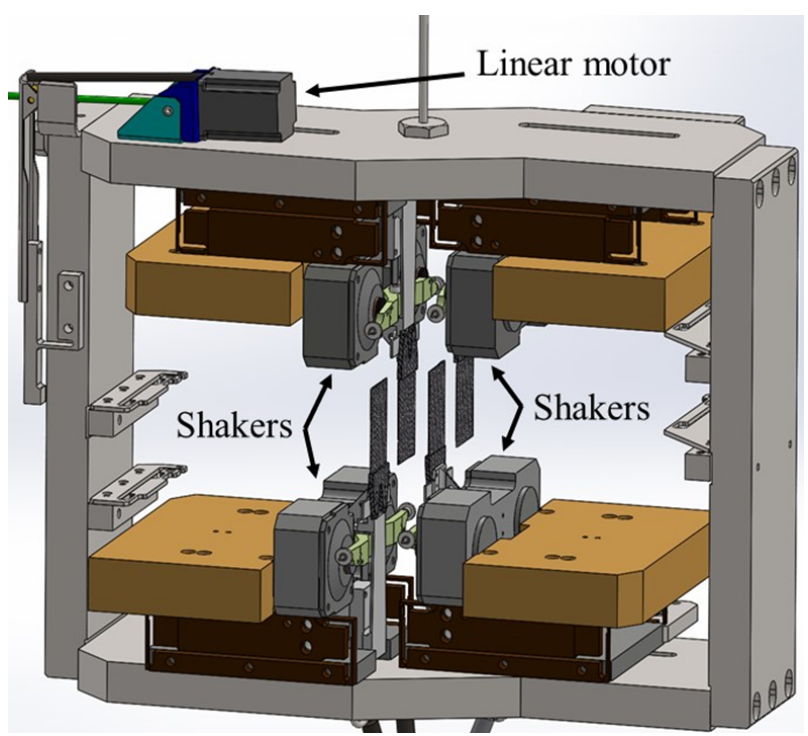

FiguRE 3. Supporting frame with four electromagnetic shakers.

Fig. 3 shows four electromagnetic shakers which are alternately attached at the top or at the bottom of the supporting frame. Motions of blades (torsion and bending) are realized by elastic suspensions of the electromagnetic shakers (Fig. 4). Main and auxiliary elastic elements are specifically designed in a way which provides bending and torsion motions. Two moving coils fixed on a cross beam are partially inserted into larger solenoids (permanent magnets). An oscillation mode is defined by a phase delay between the electric currents running through two moving coils. These electric signals are proportional to forces and moments. Each moving coil has its feedback control system in order to set a precise blade motion. Further details about the experimental setup are described in [7.

A technique developed in [9] is applied in order to measure unsteady aerodynamic forces and moments. This technique assumes a linear model of aerodynamic loading and it is suitable especially for low-amplitude vibrations. To put it simply, identical blade cascade motion is set up and measured with and without flow so that the aerodynamic loading could be estimated by the difference between these two measurements. Furthermore, there are three basic conditions which are necessary for an accurate measurement and evaluation: (i) the system operates in resonance (exciting frequency) and the natural frequency for the bending 


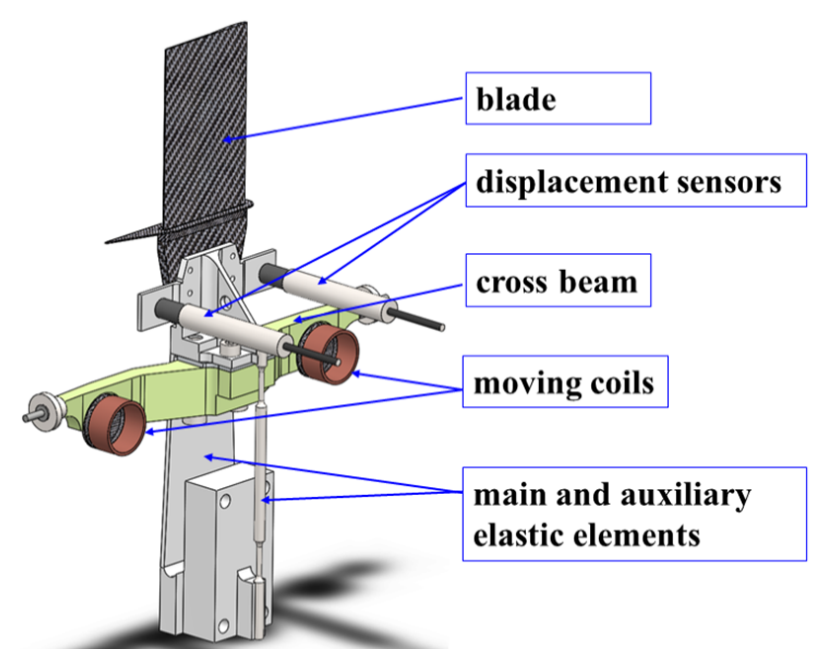

FiguRE 4. Elastic suspension.

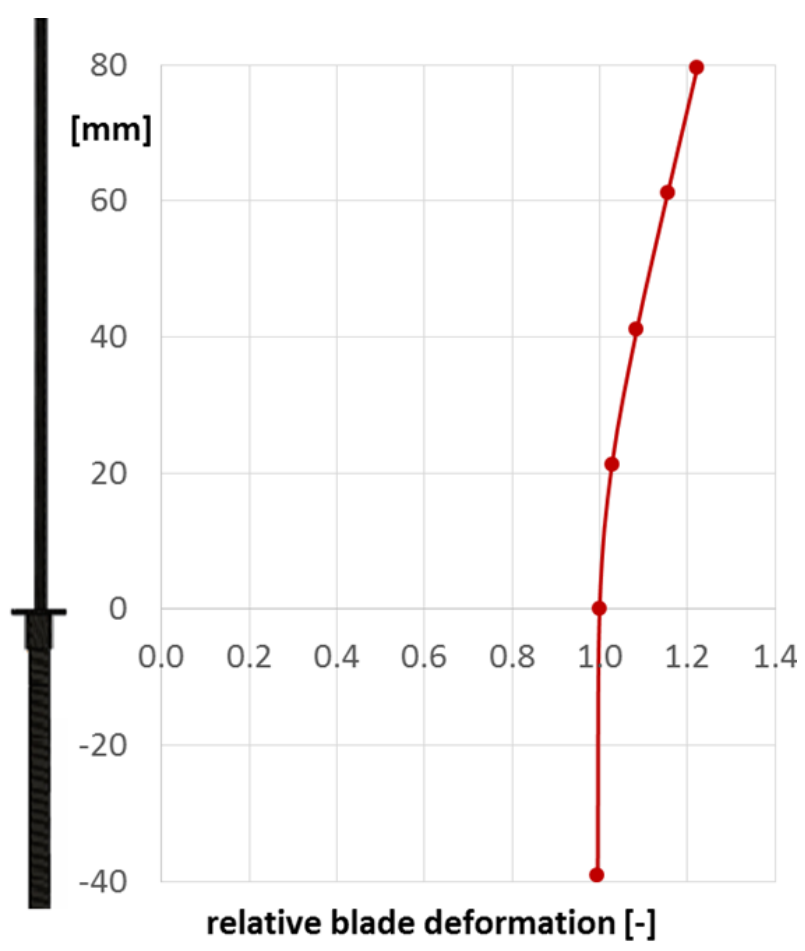

FiguRE 5. Experimentally measured blade flexible deformation in quiescent air at bending vibration.

mode and the natural frequency for the torsion mode are the same (system is tuned to $82.2 \mathrm{~Hz}$ ), (ii) the centre of the mass of the elastic suspension coincides with the axis of rotation and (iii) mechanical damping is small. Due to the fact that the blade tip is subjected to larger amplitudes than the blade root during bending vibration (Fig. 5), the blade deflection must be accounted for the analysis of unsteady aerodynamic forces and moments [10, 11]. For the test cases described in this paper, the travelling wave mode in the bending motion was defined with the vibration amplitude of $0.7 \mathrm{~mm}$ at the blade root. Similarly, torsion motion was defined with the vibration amplitude of $0.5^{\circ}$.

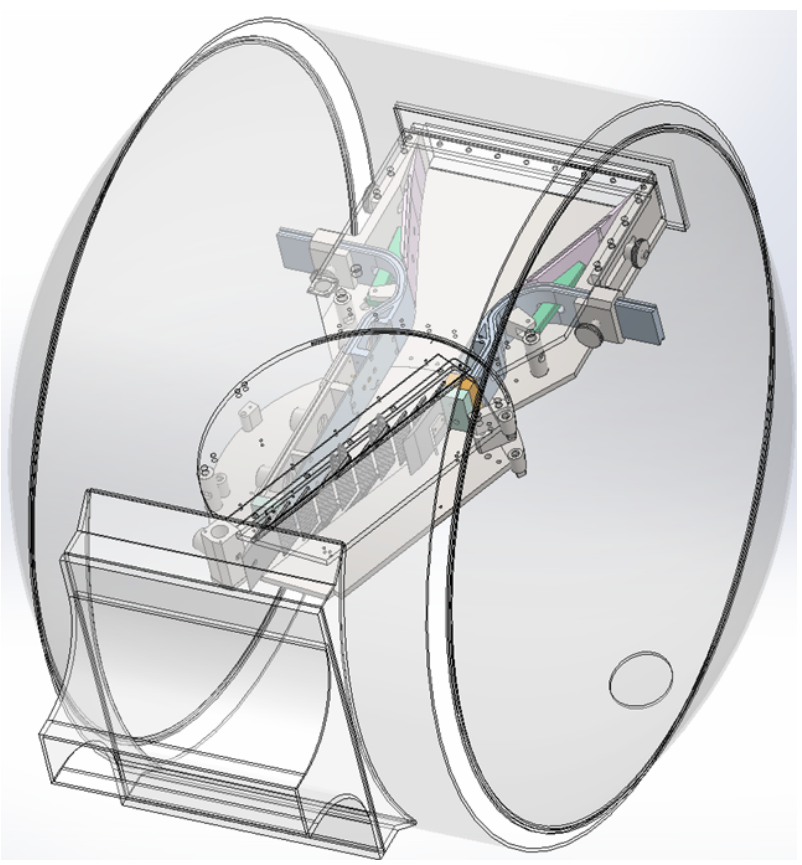

Figure 6. Original model of Experimental test section.

\section{ANSYS CFX NUMERICAL ANALYSIS}

As one of the CFD numerical tools, the commercial code ANSYS CFX 18.2, which is widely used in DSPW, has been used to carry out numerical simulations. The original model of the wind tunnel test section is shown in Fig. 6. This model has been simplified for numerical simulations based on detailed CFD investigations [12]. Final computational domains for steady-state and unsteady simulations are shown in Fig. 7. The inlet of the domains is represented by the red planes and the outlet of the domains is represented by the blue planes.

Unstructured hexahedral meshes (Fig. 8) were created in whole computational domains using ANSYS ICEM CFD 18.2. The first element height in the boundary layer was set to $1 \cdot 10^{-5} \mathrm{~m}$ which was found to be adequate for this study [13. The total number of elements for steady-state simulations is about 16 million. Because unsteady simulations were extremely time-consuming, the domain for unsteady simulations was further simplified and the computational mesh was refined to the final number of elements of 3.4 million. For example, the total CPU time on twenty-core $3.10 \mathrm{GHz}$ system was about 1 hour for one steady-state simulation. The computational time for unsteady simulations using the same machine was considerably longer. The resulting CPU time was about 16 hours for 6 periods (a number of periods which were necessary in order to achieve solution periodicity).

The flow field in the computational domain was modelled as viscous and fully turbulent. Thermodynamic properties of the medium (air) were described by the ideal gas law. The two-equation eddy-viscosity SST k- $\omega$ turbulent model with an automatic wall function was used for the turbulence modelling and second 


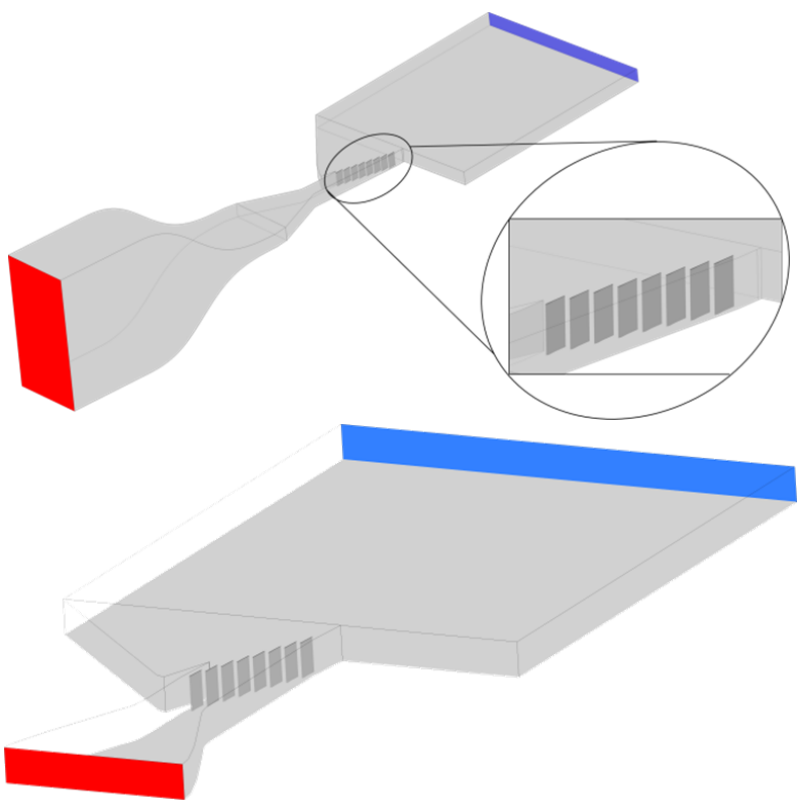

FiguRE 7. CFX computational domain for steadystate simulations (up) and unsteady simulations (down).
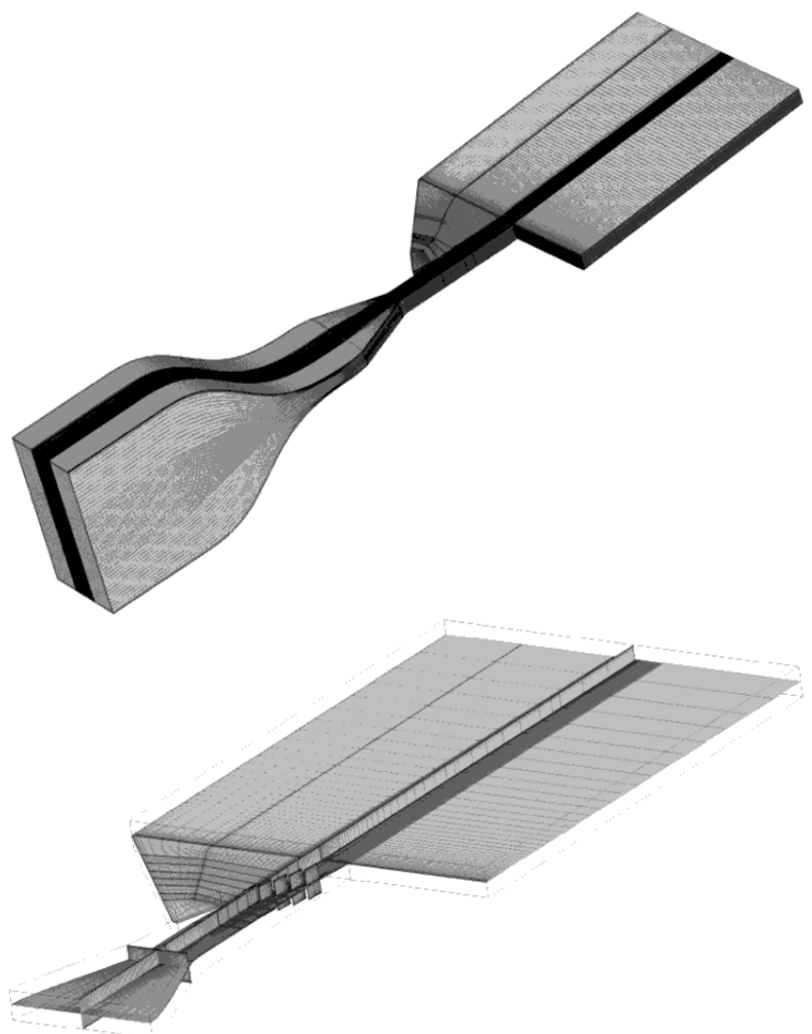

Figure 8. Computational mesh for steady-state simulations (up) and unsteady simulations (down).

order accurate approximations for all terms in the governing equations were applied in all CFX numerical simulations. The time period for the unsteady simulations was defined as $1 / 82.2 \mathrm{~s}$, which the reciprocal value of the tuned frequency $82.2 \mathrm{~Hz}$, and the total number of time steps per period was defined as 100 . The total time duration of unsteady simulations was specified using the total number of periods per run. It was investigated that 6 periods per run were sufficient enough to calculate the required aerodynamic work [7, 12. To quantify convergence criteria of steadystate simulations, the residual values were used where the RMS (root mean square) residual type was chosen and $1 \cdot 10^{-5}$ residual target value was defined. The global imbalances were less than $0.1 \%$ for all cases. For unsteady simulations, the same target residual was used in order to control the termination of iterations. The maximum number of coefficient iterations per time step was defined as 5. Since a total pressure and a static temperature are measured in the inlet, they were used as initial inlet boundary conditions. Then their values were iteratively adjusted so that the total pressure in the traverse plane downstream of the blade cascade would be approximately equal to the measured values (Fig. 9 down). The mass flow rate was defined at the domain outlet and its value was modified in a close range until the mean static pressure value in the traverse plain was approximately equal to the measured value (Fig. 9 up). For unsteady simulations, a total pressure, a total temperature and velocity vectors taken from the steady-state simulation were used as the boundary conditions at the inlet and the mass flow rate value defined at the domain outlet remained the same. Reflecting boundary conditions could be used since no issues with reflections were observed in the simulations. The blade bending motion was defined according to the measured relative blade deformation and defined amplitude. The same blade attachment was considered as in the experiment (Fig. 3). This means that the first vibrating blade (blade $\# 3$ ) is attached at the top, the second is attached at the bottom etc. The blade bending was defined as the translational motion perpendicular to the oncoming flow direction with the motion amplitude plus the blade deformation specified by a function where the independent variable is a blade length coordinate (see Fig. 5 for more details). The blade torsion motion definition is easier. It is just the motion around the rotation axis in the middle of blade chord. Before starting the unsteady simulations, the static and total pressure distributions at $50 \%$ of the blade height across the channel downstream of the blade cascade were compared with the experimental data (Fig. 9). As the numerical results are based on the adjustment of boundary conditions according to the experimental results, the agreement is good especially in the total pressure distribution. In the static pressure distribution, we can see some differences, especially in the CFX approach. In case of the TRAF code results, the domain is simplified (Fig. 10) so that the outlet static pressure can be defined as a boundary condition exactly according to the experimental data very close to the traverse plane. In case of the CFX, the domain is more complex (Fig. 7) and outlet with defined boundary condition is more distant. Furthermore, the mass flow rate is defined as the outlet boundary 

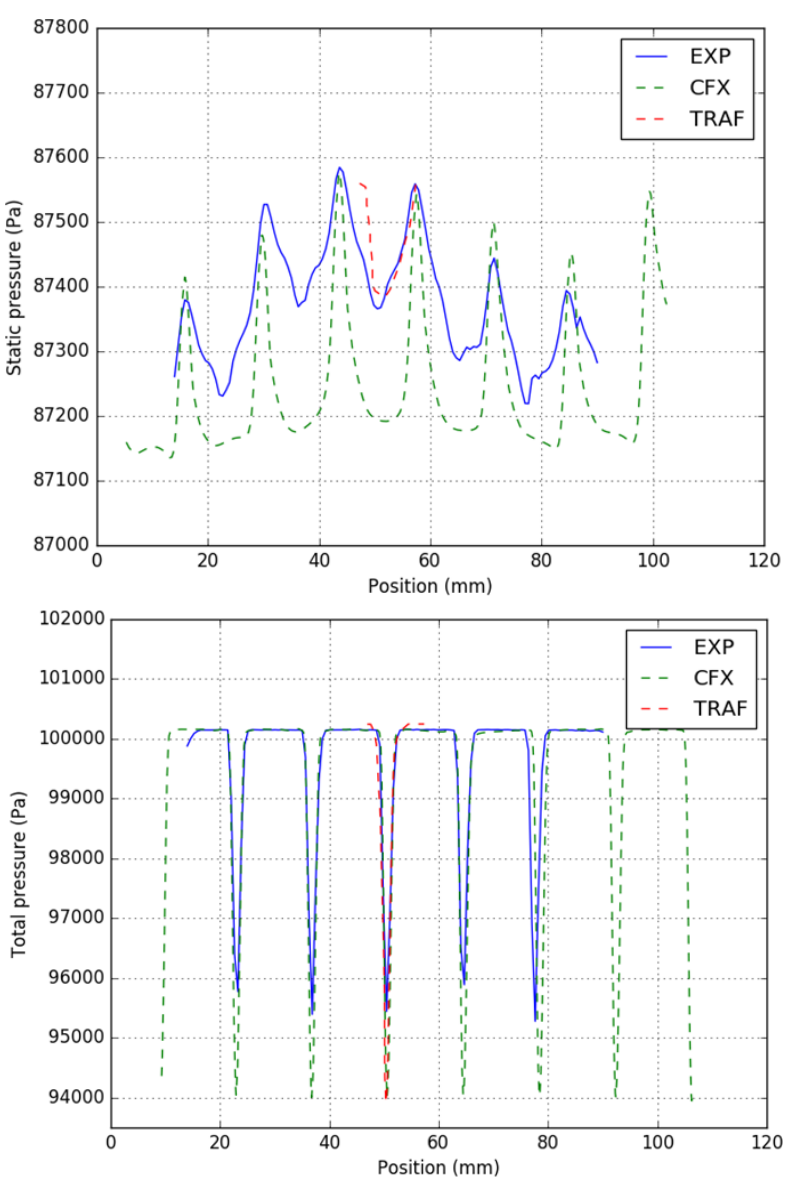

Figure 9. Comparison of the experimental and CFD data of CFX and TRAF (static pressure (up) and total pressure (down) in the traverse plane downstream of the blade cascade shown in Fig. 2 .

condition. After the sensitivity analysis of the outlet mass flow rate influence on the static pressure distribution in the traverse plane was carried out, it was concluded that all results have similar differences as in Fig. 9 and that the differences between the mean value and the peaks are supposed to be small. Therefore, no other boundary condition adjusting would be necessary. Further details of the differences caused by experimental approach are commented in [7].

\section{TRAF CODE NUMERICAL ANALYSIS}

The TRAF code is other solver employed to predict steady-state and flutter response of the UWB testrig. This is an in-house CFD solver developed at the University of Florence used for aerodynamic, aeromechanic and aeroacoustic analyses of turbomachinery environments 14. The 3D aerodynamic solver TRAF was extended to analyze unsteady flows around vibrating rows by using a re-meshing strategy, thus implementing an aeroelastic non-linear method able to deal with tuned rows, cluster systems and mistuned disks and linear cascades. The validation of the flutter procedure in subsonic and transonic tuned turbine environments can be found in 2, 3. To perform a flut-

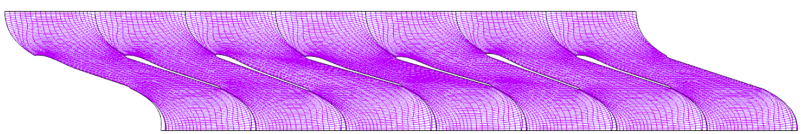

Figure 10. Simplified domain for TRAF computations.
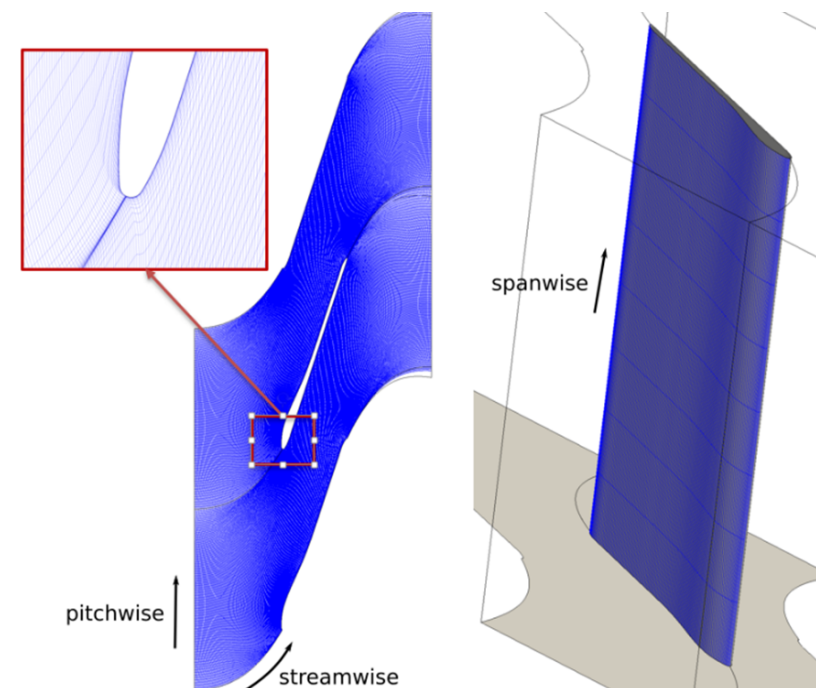

Figure 11. Details of the 3D grid.

ter analysis, time-sinusoidal blade vibrations coming from modal analysis or from vibration measurements are assigned to the unsteady calculations as input data. A re-meshing technique is used to actually deform the grid at each time step during the unsteady run. The non-linear unsteady flow equations are integrated on the deforming domain by applying the same dual time-stepping algorithm used for aerodynamic unsteady simulations. Traveling waves are solved on a single-angular-pitch annular domain by applying phase-lagged conditions to periodicity boundaries. Fourier transforms are used to assign quantities on periodicity boundaries with appropriate time shifts, limiting the memory storage requirements. It is also possible to compute blade cluster system with different blade geometries applying an arbitrary phase shift between adjacent blades within annular and linear packet [3].

For all the steady-state and flutter (unsteady) analyses, it was decided to simplify the geometry of the actual test-rig to evaluate the possibility to obtain an accurate flutter assessment with the tailored computational model. Basically the two solid walls located at both sides of the 8-blade linear cascade (clearly visible in the CFX model (see Fig. 6), were removed and replaced with periodic boundary condition. Also the inlet plenum was not included in the computational domain and all the analyses were focused on the domain shown in Fig. 10. This simplification decreases a lot the computational cost and allows a better discretization of the flow channel.

A H-type mesh was created and duplicated to reproduce the whole linear cascade. The H-type grid dimensions are $396 \times 80 \times 8$ (streamwise $\mathrm{x}$ pitchwise $\mathrm{x}$ 

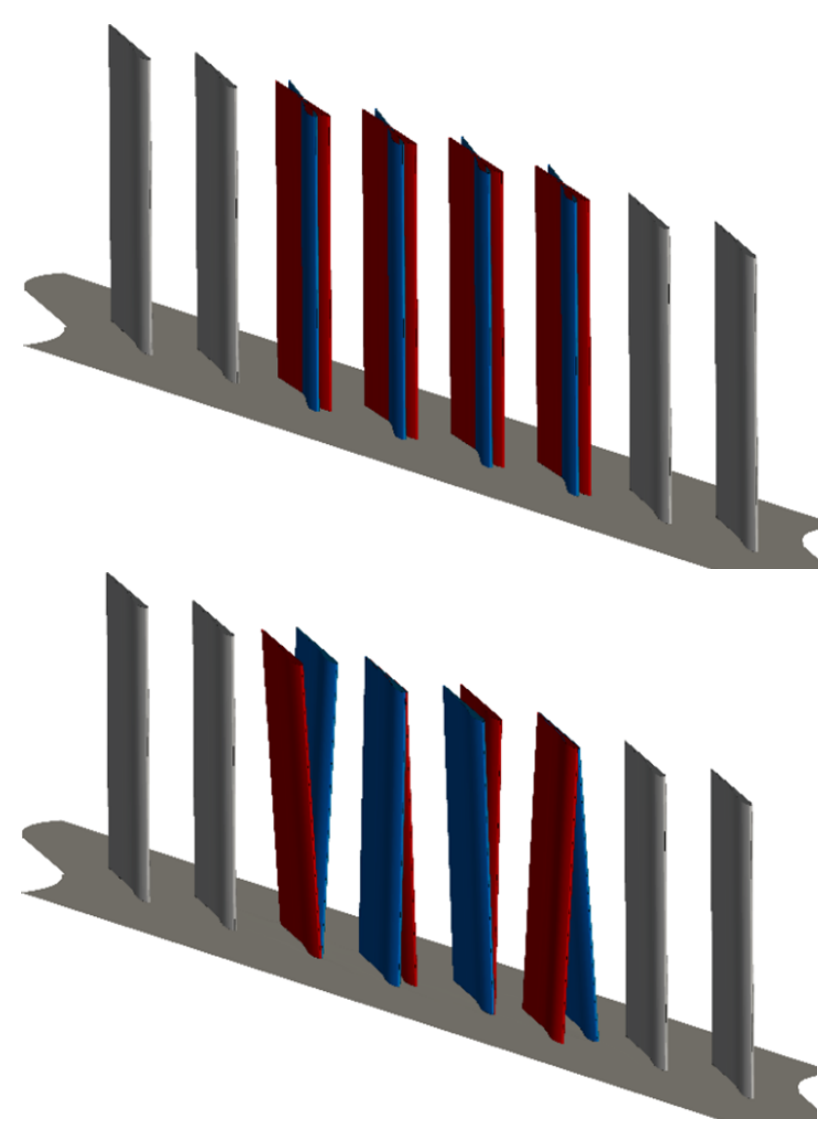

Figure 12. Torsion mode-shape and CFD domain deformation (up), bending mode-shape and CFD domain deformation (down).

spanwise, see Fig. 11 for direction definitions) and the total number of elements is about 2.5 million. As can be seen in Fig. 11, the blade to blade grid is viscous, while along the span-wise direction the mesh is inviscid. This type of mesh allows a further reduction of the computational cost, especially for the following unsteady simulations with vibrating profiles. A fine mesh with a viscous stretching in the span-wise direction was also generated for comparison purposes, the results do not differ significantly. Steady-state results were computed with TRAF code using a k-omega turbulence model. Inlet and outlet boundary conditions were provided by UWB in terms of total and static pressure, total temperature and flow angles. Steadystate results in terms of pitch-wise distributions at the outlet midspan section of total and static pressure are compared to experimental values. Since numerical simulations do not include cascade sidewalls and all the vanes are periodic, just the distribution after the central blade is included in Fig. 9 for comparisons. As it can be noticed, the agreement with the inner part of the blade cascade is very good in terms of both static and total pressure. For flutter assessment, the work exchange between vibrating profiles and the flow is evaluated by using an uncoupled non-linear flutter method implemented in the TRAF code [10, 11].

In the test rig, the 4 inner blade profiles are vibrated by mean of shakers and the flexible component of the

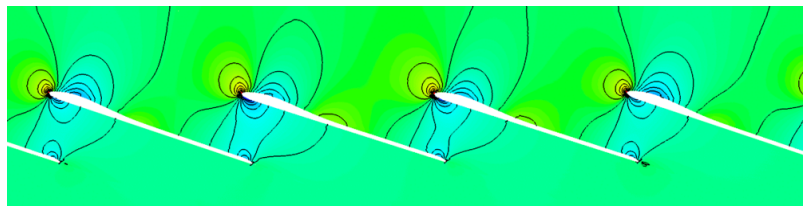

FiguRE 13. Instantaneous pressure field due to blade torsion oscillation.

suspension is alternately located under the root or above the tip of single blade, the blade modes were basically considered as "rigid" mode-shapes (bending and torsion). For this reason, the cascade oscillation imposed by the shakers can be reproduced just knowing an oscillation axis, an oscillation amplitude and the Inter Blade Phase Angle (IBPA). In order to deform the computational domain according to whole cascade oscillation, a mode shape transfer technique is used to interpolate the rigid real modes artificially built to the blade surfaces within the multi-block CFD mesh. When referring to classical flutter problems, the row oscillation is the only source of unsteadiness and, obviously, the whole fluid domain (where NavierStokes equations are numerically integrated by the solvers) has to be deformed following the blade-row vibration. The non-linear method employs a grid deformation strategy to actually rebuild the computational domain at different equally-spaced instants over the oscillation period. To avoid cell intertwining, the grid deformation is built by using an algebraic method which distributes the largest deformations where the biggest mesh elements are located, thus maintaining the shape of the smallest elements located, for instance, within the boundary layer or near the blade clearance.

Then, the non-linear unsteady flow equations are integrated on this deforming domain by applying the same dual time-stepping algorithm used for aerodynamic unsteady simulations. The computations are iterated until the unsteady pressure on the profile surfaces due to blade oscillation becomes periodic (see instantaneous static pressure in Fig. 13). Finally, once the solution is periodic, the aerodynamic work (Eq. 1) can be computed during one blade oscillation period. Since in uncoupled methods blade vibrations are input data, rather than a result of the computation, flutter stability is estimated by checking the sign of the aerodynamic work done by the fluid onto the blade during one vibration cycle as explained in the following section. The first vibrating test-case, which has been analyzed, is characterized by 4 inner blades moving of rigid torsion with different IBPAs. The torsion oscillation axis is imposed at the middle of the profile chord and the oscillation angle is $\alpha=0.5^{\circ}$. As it can be seen in Fig. 12 up, the actual mode-shape of the blade and the suspension system shows a rigid torsion vibration of the blade surfaces. This aspect confirms the rigid mode approximation hypothesis used herein for all the unsteady analyses. 


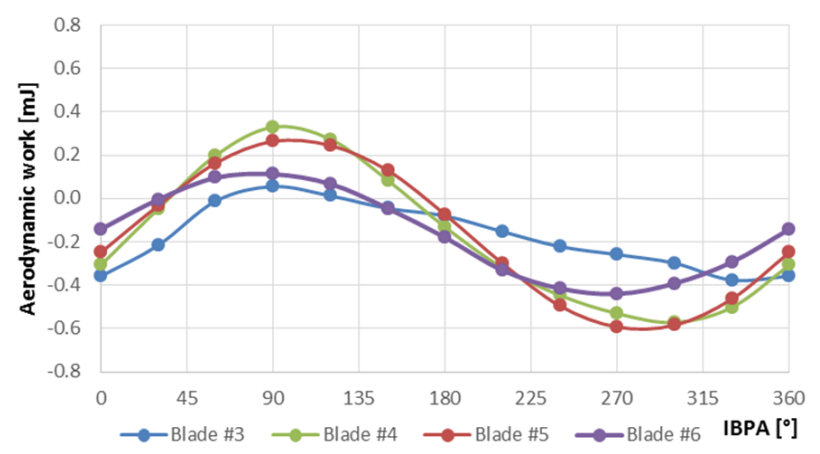

FiguRE 14. Blade torsion: aerodynamic work for the 4 vibrating profiles vs. IBPAs.

These rigid mode-shapes were transferred to the blade surface within the CFD mesh and, for each IBPA a different mesh deformation was created. Fig. 12 down shows on the bottom the $3 \mathrm{D}$ view of CFD domain with the moving blades in the two extreme positions during the oscillation cycle. Once the grid deformation was generated, URANS computations with the vibrating cascade were run. The steady-state solution, already computed, is used as flow initialization and the unsteady analyses are iterated until the periodicity is reached. Usually $5 / 6$ blade oscillation periods are required for periodicity and a further period is run to compute the aerodynamic work and to extract unsteady forces on the vibrating blades. The aerodynamic work is computed by the solver while running the last period over each vibrating profile for each IBPA. The aerodynamic work curves for torsion testcase are shown in Fig. 14. As it can be seen, the aerodynamic work curves have a typical sinusoidal trend and, some IBPAs show positive aerodynamic work and so flutter instability, as expected. There is an excellent agreement between numerical and experimental data as shown in the following section. This suggests that the simplified setup used for the flutter computations is suitable to evaluate the flutter assessment of the linear cascade.

The second test-case is dedicated to blade bending motion. As for the torsion test-case, the blade oscillation is approximated with a rigid mode and no blade deformation is modeled. The oscillation axis is oriented in the direction of the blade chord and for the sake of simplicity, the pivot point is located below the blade root. The blade oscillation systems are installed in the rig at the hub and tip of the channel alternately. A 3D view of blade deformation in two extreme positions for IBPA $=60^{\circ}$ is depicted in Fig. 12 down. As for the torsion test-case, the unsteady simulations were performed starting from steady-state flow solutions. Again, when the periodicity is reached, aerodynamic work and unsteady blade forces were extracted. These bending test-case points out an overall stability of the cascade (see Figs. 18 . 18 where all the profiles for each IBPA have a negative value of aerodynamic work, meaning that the energy goes from the blade to the fluid and the system is damped). As it is shown in the following section, the agreement with experimental data and numerical result with CFX is good confirming the applicability of domain approximations already described.

\section{AERODYNAMIC WORK DEFINITION}

The aerodynamic work per cycle done by the fluid on the blade per vibration cycle was numerically computed as follows in Eq. 1 [3]:

$$
A W_{\text {cycle }}=\int_{t_{0}}^{t_{0}+T} \int_{A}-p \mathbf{V n} d A d t
$$

where $T$ is the period of one vibration cycle, $t_{0}$ is the time at the start of the vibration cycle, $p$ is the instantaneous fluid pressure, $\mathbf{V}$ is the local velocity of the blade surface due to imposed vibrational displacement (a defined blade motion), $A$ is the surface of the blade and $\mathbf{n}$ is the unit normal vector to the surface $A$. If the aerodynamic work per cycle done on the blade is positive, the work is being imparted to the blade, generating negative damping, which indicates a potentially unstable situation leading to a self-sustained vibration (flutter) likely to cause a material fatigue failure. On the other hand, if the aerodynamic work per cycle done on the blade is negative, the blade motion is doing work on the fluid and leads to a stable or damped vibration. In general cases, it is necessary to evaluate some dimensionless parameter such as a logarithmic decrement, a damping ratio or a critical damping ratio [4 6], because the value of vibration amplitude affects the results of the aerodynamic work. The vibration amplitude is in real cases (real turbine rotor blades) different than it is defined in numerical simulations. However, in this study, the vibration amplitude is exactly defined in the experimental measurement so that the same value can be defined in the numerical analyses. Therefore, the aerodynamic work can be directly compared. Further details can be found in [9]1].

\section{Bending MOtion Results COMPARISON}

The aerodynamic work expressed in Eq. 1 is the main quantity to assess flutter stability and was experimentally and numerically evaluated for all the IBPAs of the two vibration test cases (i.e. bending and torsion) at $\mathrm{Ma}=0.42$ and $\mathrm{AOA}=0^{\circ}$. Figs. 15 18 show the comparisons of the aerodynamic work in the bending motion with the vibration amplitude of $0.7 \mathrm{~mm}$ for each flexibly mounted blade. The data scatter between three sets of data (experiment - EXP and two numerical simulations - CFX and TRAF) is obvious, both numerical results may differ by more than $100 \%$ in comparison with the experimental measurements, however blade cascade stability has been found in all results. The main reason for the discrepancy in the 


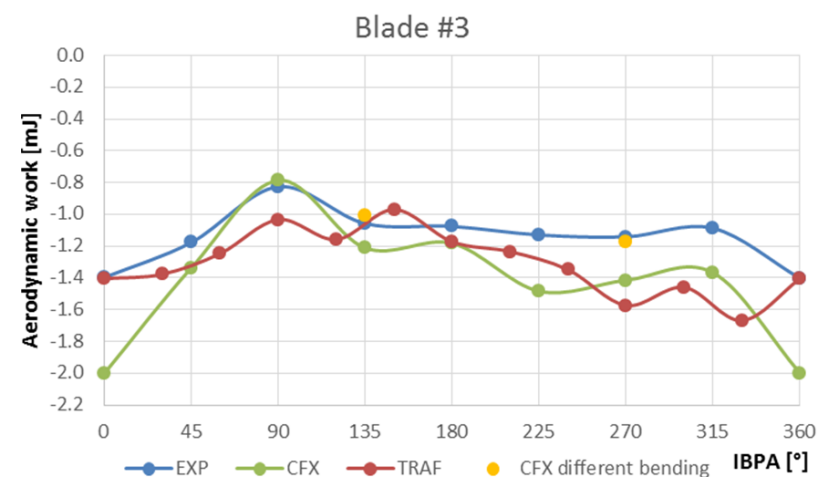

Figure 15. Aerodynamic work of blade bending motion - blade \#3: experimental (EXP), CFX and TRAF comparison.

results can be found in the difference of blade deformation in bending motion with and without flowing air. While the blade bending deformation in quiescent air (see Fig. 57 was easily measured, as the supporting frame with the shakers (see Fig. 3) was placed outside the test chamber, and the data was employed in CFX numerical simulations, a true bending deformation in flowing air could not be obtained experimentally. It is expected that the flow will significantly affect the blade bending deformation and therefore experimental and numerical results must differ. In order to confirm the model performance sensitivity on a blade bending definition, a new bending motion without the blade deformation was carried out in CFX for IBPAs $135^{\circ}$ and $270^{\circ}$. These results are also shown in Figs. 15, 18 (CFX different bending) and they disagree with the previous solutions. The fact that they are closer to the measured values might suggest that the flow has a tendency to reduce the blade bending deformation. However, such a conclusion is immature due to the lack of data and the issue can be investigated further. Moreover, simplifications of the definition of the bending motion in the TRAF code (i.e. rigid blade with the pivot point, which is located below the blade root, in reality, considerably below the blade root) also contribute to the disagreement between the experiment and computational data. Therefore, the experimental and the numerical results show different blade bending motions and the discrepancies in the results in Figs. 15 18 are correct. Further work is expected to improve the absolute accuracy of the predictions and the measurements. This will be necessary for situations, where aerodynamic instability in bending motion can occur (i.e. testing at different angles of attack, lower (reduced) frequencies or coupled bending-torsion motions). Anyway, it is important to pinpoint that the aerodynamic work for the bending configuration in this particular test case is always negative for each blade and this aspect was correctly captured by both numerical methods despite the discrepancies in blade oscillation definition. This bending configuration at $\mathrm{Ma}=0.42$ and $\mathrm{AOA}=0^{\circ}$ shows an overall stable response with no danger of flutter occurrence.

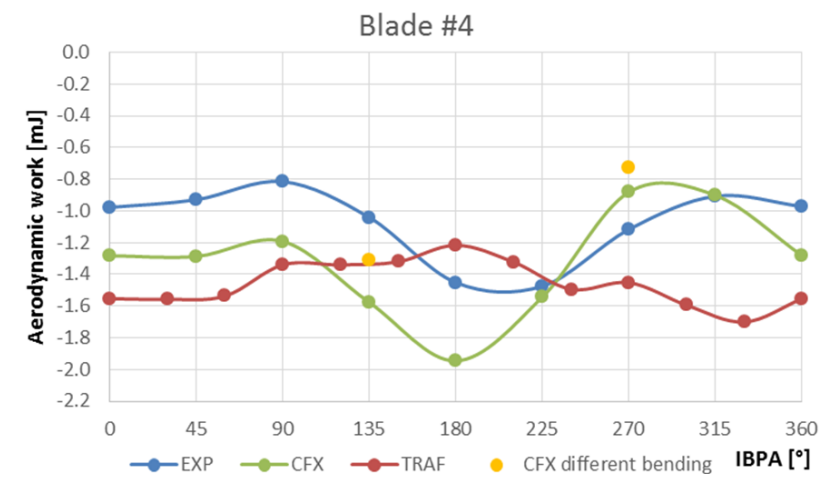

Figure 16. Aerodynamic work of blade bending motion - blade \#4: experimental (EXP), CFX and TRAF comparison.

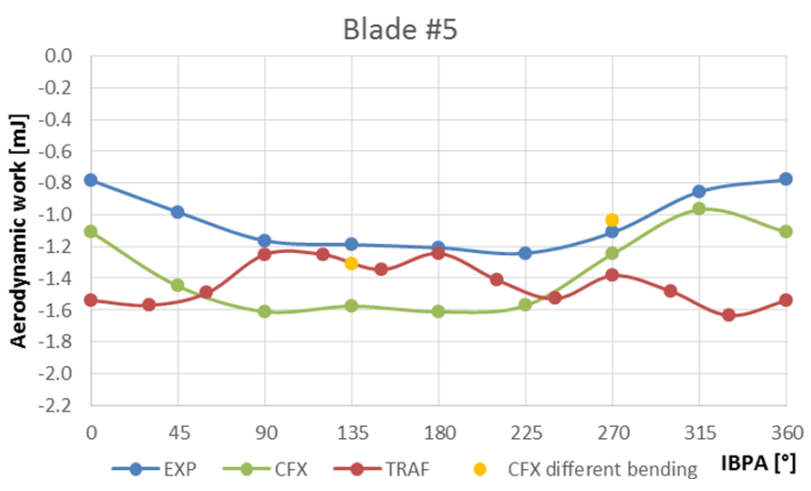

FIGURE 17. Aerodynamic work of blade bending motion - blade \#5: experimental (EXP), CFX and TRAF comparison.

\section{TORSION MOTION RESULTS COMPARISON}

Figs. 19.22 show the comparisons of aerodynamic work curves as a function of the IBPAs for the flexibly mounted blades 3-6 in the case of blade torsion with the vibration amplitude of $0.5^{\circ}$ at $\mathrm{Ma}=0.42$ and $\mathrm{AOA}$ $=0^{\circ}$. Contrary to the blade bending motion test case, the discrepancies in terms of aerodynamic work are reasonably small and the agreement between all the curves is more than satisfactory for each blade. This agreement can be achieved due to the fact that the torsional motion is better defined than the bending case. Although no evident blade deformation was measured in quiescent air for the torsion motion, flowing air can change slightly the shape of oscillating blade, however this effect cannot be experimentally observed. Such a torsional deformation of blade might also contribute to the small discrepancy between the experiments and the numerical simulations. It can be noticed that there is a certain range of positive IBPAs where the aerodynamic work is positive, thus highlighting a flutter instability for all the vibrating profiles. Both codes delivered similar values of energy exchange, but in the direct comparison to experimental data, the range of unstable IBPAs is better predicted by CFX. In this test case for torsion motion at $\mathrm{Ma}=0.42$ and $\mathrm{AOA}=$ $0^{\circ}$, both computational models have been successfully 


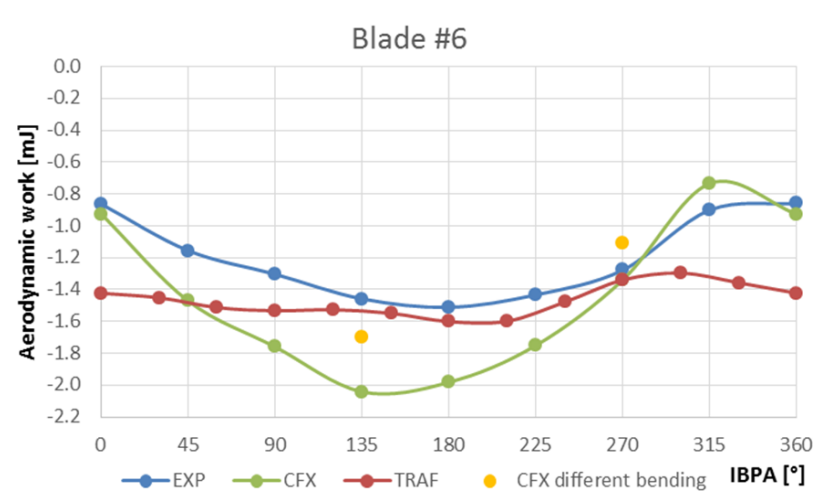

Figure 18. Aerodynamic work of blade bending motion - blade \#6: experimental (EXP), CFX and TRAF comparison.

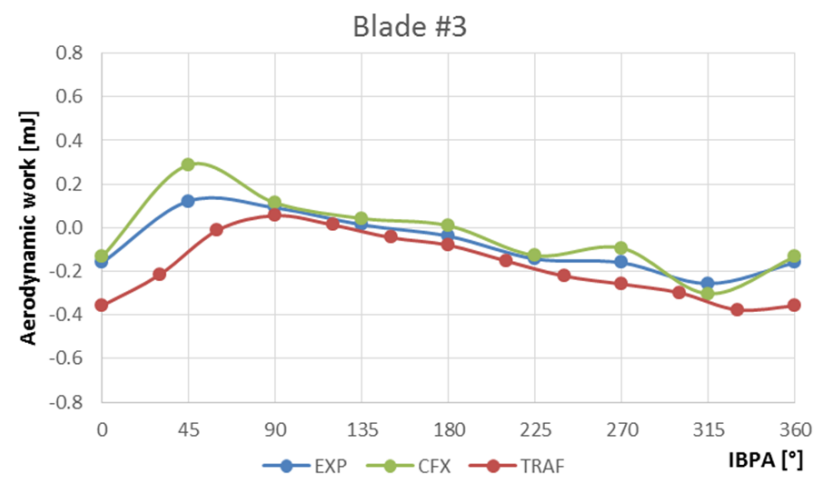

Figure 19. Aerodynamic work of blade torsion motion - blade \#3: experimental (EXP), CFX and TRAF comparison.

validated and proved suitability for flutter simulations of slender blade profiles.

\section{Conclusions}

Experimental measurements of the flutter behaviour of model of LSB were carried out using an in-draft wind tunnel where a turbine cascade with slender blades in a linear configuration is installed. Four inner blades of the cascade were flexibly mounted each with two degrees of freedom to generate bending and torsion motion. Two different numerical methods were applied for the flutter assessment of this blade cascade: a commercial code (ANSYS CFX) and an in-house code (TRAF). In each method, a rig geometry model with different level of complexity was used. A travelling wave mode for bending motion with vibration amplitude of $0.7 \mathrm{~mm}$ and torsion motion with the vibration amplitude of $0.5^{\circ}$ was investigated at $\mathrm{Ma}=$ 0.42 and $\mathrm{AOA}=0^{\circ}$. To some extent, very good agreement between all compared cases has been achieved. In detail, the bending test case showed discrepancies between the numerical and experimental aerodynamic work values. This can be explained by different blade oscillation behaviour (due to the blade flexibility) during the experimental campaign with respect to the blade oscillation imposed for the numerical computations. However, the overall stability of blade cascade

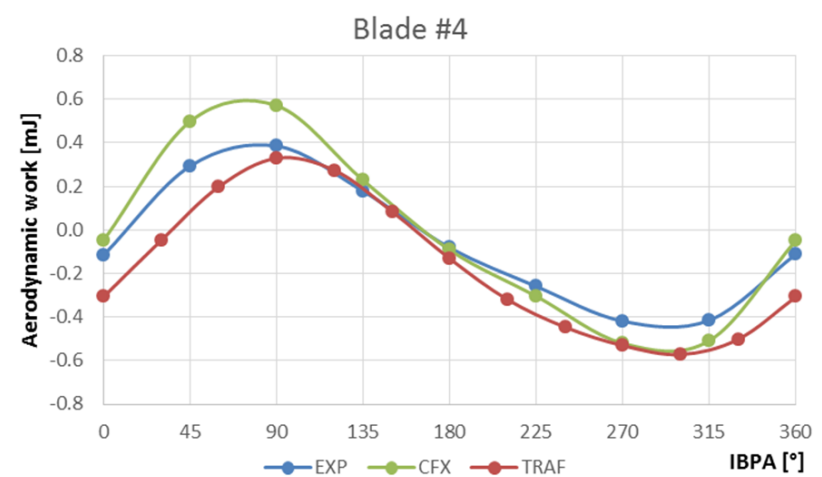

Figure 20. Aerodynamic work of blade torsion motion - blade \#4: experimental (EXP), CFX and TRAF comparison.

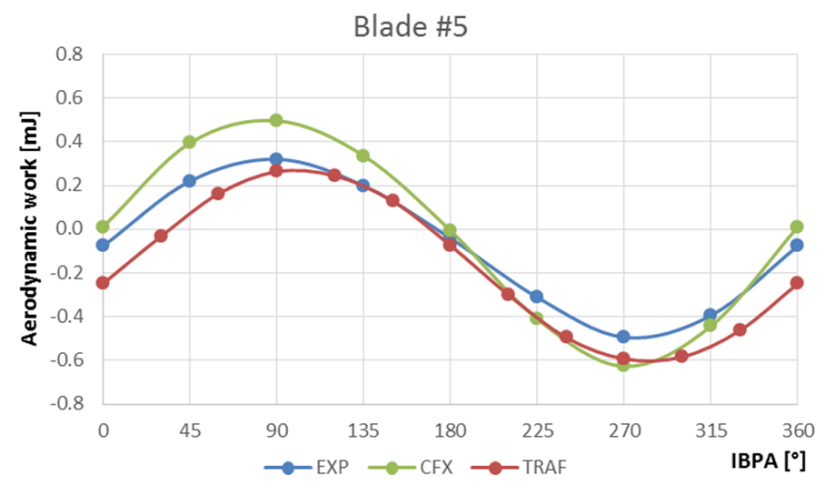

Figure 21. Aerodynamic work of blade torsion motion - blade \#5: experimental (EXP), CFX and TRAF comparison.

has been observed using both numerical approaches and also in the experiments. On the other hand, the torsion test case, where the blade shape is not so modified by the shakers (but to a lesser extent by the flow), shows very good agreement between the experimental data and the numerical results. The multi-purpose commercial code CFX confirms its flexibility in simulating aeromechanical investigations of complex geometries (including sidewalls and the actual domains up/downstream the blade cascade). On the other hand, the in-house code TRAF is basically conceived to internal flow simulations for turbomachinery environments and a few approximations in the geometry discretization are required. Yet, the TRAF code suite takes advantage of low computational cost, the possibility of a better discretization of the flow channel, an easy and quick mesh deformation strategy and of dedicated tools for flutter post-processing which speed up typical flutter analyses. The results in this paper confirm that the used flutter tools are capable to predict aerodynamic work exchange between fluid and vibrating profiles in air flows. These tools can be now widely tested for multi-phase flows and subsequently used during the design phase, especially in off-design conditions required by flexible operations to avoid HCF failure due to flutter vibrations. 


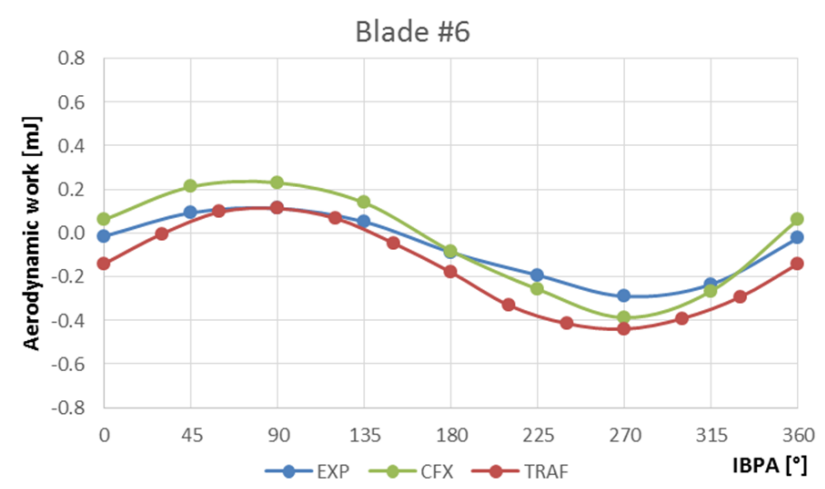

Figure 22. Aerodynamic work of blade torsion motion - blade \#6: experimental (EXP), CFX and TRAF comparison.

\section{ACKNOWLEDGEMENTS}

This research effort was founded by the European project Flexturbine, H2020 research and innovation programme under grant agreement No. 653941.

\section{REFERENCES}

[1] T. Tanuma. Advances in Steam Turbines for Modern Power Plants. Woodhead Publishing, 2017. https: //doi.org/10.1016/B978-0-08-100314-5.00001-4

[2] L. Pinelli, F. Poli, A. Arnone, C. Schipani. A Time-Accurate 3D Method for Turbomachinery Blade Flutter Analysis. 12th International Symposium on Unsteady Aerodynamics, Aeroacoustics and Aeroelasticity of Turbomachines (ISUAAAT), London, UK, 2009. Paper I12-S8-3, http://arnone.de.unifi.it/tgroup/node/891.

[3] F. Vanti, L. Pinelli, F. Poli, A. Arnone. Aeroelastic Investigation of Turbine Blade Assemblies: Cluster System and Mistuned Rows. 12th European Conference on Turbomachinery Fluid Dynamics \&

Thermodynamics ETC12, 2017. https://doi.org/10.29008/ETC2017-144

[4] C. Fuhrer, D. M. Vogt. On the Impact of Simulation Approaches on the Predicted Aerodynamic Damping of a Low Pressure Steam Turbine Rotor. ASME Turbo Expo, 2017. https://doi.org/10.1115/gt2017-63401

[5] T. Sun, P. P. Repar, Q. Di. Investigation of Tip Clearance Flow Effects on an Open 3D Steam Turbine Flutter Test Case. ASME Turbo Expo, 2017. https://doi.org/10.1115/gt2017-64021.
[6] F. Vanti, L. Pinelli, A. Arnone, et al. Aeroelastic Optimization of an Industrial Compressor Rotor Blade Geometry. ASME Turbo Expo, 2018. https://doi.org/10.1115/gt2018-76474.

[7] V. Slama, B. Rudas, J. Ira, et al. The validation of flutter prediction in a linear cascade of non-rigid turbine blades. ASME Turbo Expo, 2018. https://doi.org/10.1115/gt2018-75502.

[8] I. McBean, K. Hourigan, M. Thompson, L. F. Prediction of Flutter of Turbine Blades in a Transonic Annular Cascade. ASME J. Fluids Eng., 2005. https://doi.org/10.1115/1.2060731

[9] V. Tsimbalyuk. Method of measuring transient aerodynamic forces and moments on a vibrating cascade. Strength Mater, 1996. 28: 150, https://doi.org/10.1007/bf02215842.

[10] V. Tsymbalyuk, J. Linhart. Corrections of aerodynamic loadings measurement on airfoil cascade at bending-torsion vibrations. 19th International conference on force, mass \& torgue. Cairo: National Institute of Standards Egypt, 2005. ISBN: 953-7124-00-2.

[11] V. Tsymbalyuk, J. Linhart. Corrections of aerodynamic loadings measurement on vibrating airfoils. XVII IMEKO World Congress, Dubrovnik, Croatian Metrology Society, 2003. ISBN: 953-7124-00-2.

[12] V. Slama, B. Rudas, J. Ira, et al. CFD prediction of flutter of turbine blades and comparison with an experimental test case. AEaNMiFMaE-2018, MATEC Web of Conferences 168, 2018. https://doi.org/10.1051/matecconf/201816802005

[13] J. D. Denton. Some Limitations of Turbomachinery $C F D$. ASME Turbo Expo, Power for Land, Sea, and Air, Volume 7: Turbomachinery, Parts A, B, and C: 7, 2010. https://doi.org/10.1115/gt2010-22540

[14] M. Giovannini, M. Marconcini, A. Arnone, F. Bertini. Evaluation of Unsteady Computational Fluid Dynamics Models Applied to the Analysis of a Transonic High-Pressure Turbine Stage. Proceedings of the Institution of Mechanical Engineers, Part A: Journal of Power and Energy, 2014. https://doi.org/10.1177/0957650914536170 